\title{
Lower Limit of Quantitation
}

National Cancer Institute

\section{Source}

National Cancer Institute. Lower Limit of Quantitation. NCI Thesaurus. Code C82589.

The lowest value in a possible range of values for a particular assessment. 